\title{
Onkologie
}

\section{Leichte palliative Sedierung nicht lebensverkürzend}

Maltoni $M$ et al. Palliative sedation therapy does not hasten death: results from

a prospective multicenter study. Ann Oncol. 2009;20:1163-1169

\section{Hintergrund}

Eine palliative Sedierung bedeutet die Reduzierung des Bewussteinsniveaus des Patienten mit dem Ziel, unerträgliche Symptome und Belastungen zu lindern. Inwieweit dadurch eine sekundäre Lebenszeitverkürzung resultiert, wird kontrovers diskutiert (,terminal sedation“, „slow euthanasia“) $[2,3]$.

\section{Methodik}

Eine italienische Arbeitsgruppe verglich in einer nicht randomisierten, prospektiven Multicenterstudie eine Gruppe von Tumorpatienten (Durchschnittsalter 70 Jahre) in der terminalen Phase bei weit fortgeschrittenen metastasierten Tumoren, die eine palliative Sedierung bei entsprechender Indikation $(n=267)$ erhielten, mit einer Kontrollgruppe von Tumorpatienten, die eine Routinebehandlung nach allgemeinen Krankenhausstandards erhielten $(\mathrm{n}=251)$.

$55 \%$ der Patienten hatten einen Karnofsky-Index von 20-30\%. Die drei häufigsten Primärtumorerkrankungen waren Bronchialkarzinom (19,9\%), kolorektale Tumoren $(14,1 \%)$ und Magenkarzinome $(13,1 \%)$.
Die Hauptindikationen für die Einleitung einer palliativen Sedierung waren Delir/Agitation (78,7\%), Dyspnoe (19,5\%), psychischer Stress (18,7\%) und Schmerzen (11,2\%). Bei 33\% der Patienten lagen zwei Indikationen vor.

Zur palliativen Sedierung wurden Neuroleptika (84,2\%), Benzodiazepine $(54,3 \%)$ und Opioide $(25,5 \%)$ eingesetzt. Eine Kombinationstherapie erhielten 32,6\% der Patienten und eine Sedierung mit drei Wirkstoffklassen 16,9\% der Patienten. Die mittleren Dosierungen der fünf am häufigsten verwendeten Wirkstoffe betrugen:

_Lorazepam: 4,9 mg/d (37,8\%),

- Chlorpromazin (nicht mehr im Han-

del): $55,8 \mathrm{mg} / \mathrm{d}(37,8 \%)$,

-Morphin: 41,2 mg/d (25,5\%),

- Promethazin: 49,3 mg/d (23,6\%),

_Haloperidol: 3,6 mg/d (22,8\%).

Die Dauer der palliativen Sedierung betrug durchschnittlich vier Tage bei überwiegend milder Sedierung (62,2\%). Eine intermittierende palliative Sedierung erfolgte bei 56,2\% der Patienten. Die kontinuierliche Form der palliativen Sedierung wurde mit $43,8 \%$ etwas weniger häufig eingesetzt.

\section{Überleben mit und ohne palliative Sedierung}

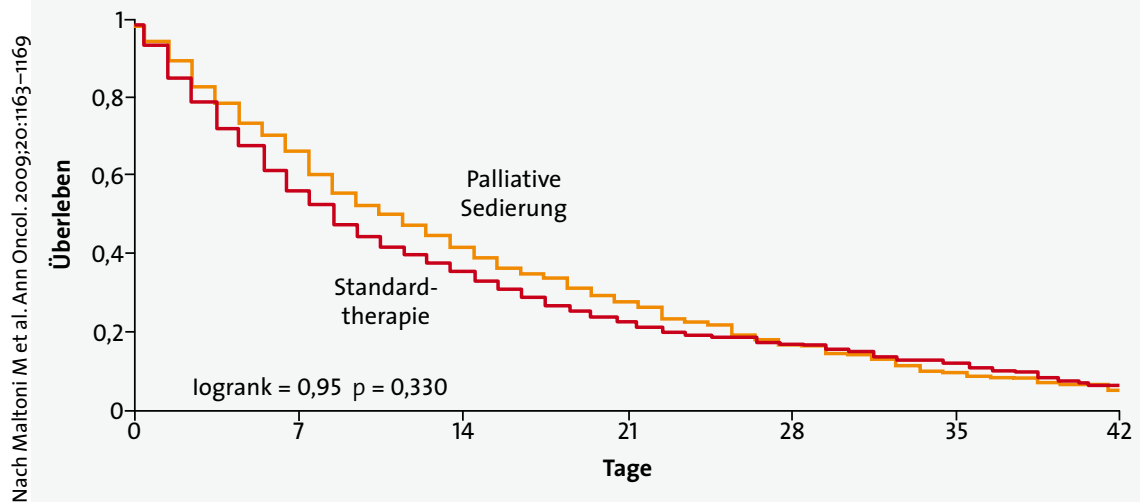

\section{Ergebnis}

Die Kaplan-Meier-Kurve ergab keinen signifikanten Unterschied im Gesamtüberleben zwischen beiden Gruppen ( $\mathrm{p}=0,330$; Abb. 1).

\section{Kommentar}

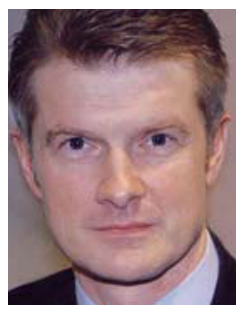

Prof. Dr. med.

Martin Kohlhäufl, Stuttgart-Gerlingen

$\mathrm{Zu}$ beachten ist, dass die überwiegende Zahl der Tumorpatienten leicht sediert wurde. Der Effekt einer raschen tiefen Sedierung auf die Überlebenszeit von Tumorpatienten bei schwerstgradiger unbehandelbarer Symptomatik kann durch diese Studie nicht beantwortet werden. Eine randomisierte Studie dazu ist ethisch nicht vertretbar. Grundsätzlich müssen die bekannten Komplikationen einer Sedierung (Atemdepression, Aspiration, Hypotonie) berücksichtigt werden, die in dieser Studie nicht systematisch erfasst wurden.

\section{Fazit}

Patienten und Angehörige können darüber informiert werden, dass eine milde Sedierung nicht lebensverkürzend wirkt.

Aktuelle Empfehlungen zur palliativen Sedierung sind publiziert $[4,5]$. Die ausführliche Diskussion im palliativmedizinischen Behandlungsteam und die Beratung von Patienten und Angehörigen bleibt eine notwendige Voraussetzung, um sicherzustellen, dass die Wünsche und Vorstellungen von Patienten und Angehörigen angemessen berücksichtigt werden und keine unethischen Gründe zu einem Missbrauch der palliativen Sedierung führen können [1].

\section{Literatur}

1. Aulbert E, Nauck F, Radbruch L (Hrsg.). Lehrbuch der Palliativmedizin. Schattauer, Stuttgart, New York, 2008

2. Ten Have H, Clark D (Hrsg.). The ethics of palliative care: European perspectives. Buckingham, UK: Open University Press, 2002

3. Lo B, Rubenfeld G. JAMA 2005;294:1810-1816

4. Higgins PC, Altilio T. J Soc Work End Life Palliate Care 2007:3:3-30

5. Veteran Health Administration National Ethics Committee. Am J Hosp Palliate Care 2006;23:483491 